\title{
Current-voltage curves of gold quantum point contacts revisited
}

Hansen, K.; Nielsen, S K.; Brandbyge, Mads; Lægsgaard, E.; Stensgård, I.; Besenbacher, Flemming

Published in:

Applied Physics Letters

Link to article, DOI:

10.1063/1.127093

Publication date:

2000

Document Version

Publisher's PDF, also known as Version of record

Link back to DTU Orbit

Citation (APA):

Hansen, K., Nielsen, S. K., Brandbyge, M., Lægsgaard, E., Stensgård, I., \& Besenbacher, F. (2000). Currentvoltage curves of gold quantum point contacts revisited. Applied Physics Letters, 71(5), 708-710.

https://doi.org/10.1063/1.127093

\section{General rights}

Copyright and moral rights for the publications made accessible in the public portal are retained by the authors and/or other copyright owners and it is a condition of accessing publications that users recognise and abide by the legal requirements associated with these rights.

- Users may download and print one copy of any publication from the public portal for the purpose of private study or research.

- You may not further distribute the material or use it for any profit-making activity or commercial gain

- You may freely distribute the URL identifying the publication in the public portal

If you believe that this document breaches copyright please contact us providing details, and we will remove access to the work immediately and investigate your claim 


\title{
Current-voltage curves of gold quantum point contacts revisited
}

\author{
K. Hansen ${ }^{\text {a) }}$ and S. K. Nielsen \\ CAMP and Institute of Physics and Astronomy, University of Aarhus, DK 8000 Aarhus C, Denmark \\ M. Brandbyge \\ Mikroelektronik Centret (MIC), Technical University of Denmark, Building 345 East, \\ DK 2800 Lyngby, Denmark \\ E. Lægsgaard, I. Stensgaard, and F. Besenbacher \\ CAMP and Institute of Physics and Astronomy, University of Aarhus, DK 8000 Aarhus C, Denmark
}

(Received 1 February 2000; accepted for publication 30 May 2000)

We present measurements of current-voltage $(I-V)$ curves on gold quantum point contacts (QPCs) with a conductance up to $4 G_{0}\left(G_{0}=2 e^{2} / h\right.$ is the conductance quantum) and voltages up to $2 \mathrm{~V}$. The QPCs are formed between the gold tip of a scanning tunneling microscope and a $\mathrm{Au}(110)$ surface under clean ultra-high-vacuum conditions at room temperature. The $I-V$ curves are found to be almost linear in contrast to previous reports. Tight-binding calculations of $I-V$ curves for oneand two-atom contacts are in excellent agreement with our measurements. On the other hand, clearly nonlinear $I-V$ curves are only observed when the sample has been cleaned in air. (C) 2000 American Institute of Physics. [S0003-6951(00)02530-4]

With the ongoing quest of miniaturizing electronic components, there is an increasing interest in fabricating and controlling atomic-scale quantum devices such as metallic quantum point contacts (QPCs). ${ }^{1-4}$ In this realm of reduced size and dimensionality, exciting phenomena can be observed. For Au QPCs, these include conductance quantization $^{2}$ and the formation of stable, single atom chains. ${ }^{3,4}$

For QPCs, the main focus has so far been on the low bias voltage regime $(V<0.25 \mathrm{~V})$ of the conductance. For high-bias, important questions concerning nonlinear conductance, ${ }^{2,5-7}$ electromigration, ${ }^{8}$ and heating arise. At the present stage, however, the available experimental results ${ }^{2,5,8,9}$ on finite bias conduction in Au QPCs are contradictory, and the theoretical interpretation of the results is unclear. $^{5-7}$ Sakai and co-workers ${ }^{8}$ studied conductance histograms acquired from breaking Au contacts in a relay and found that the $1 G_{0}$ peak did not change its position as the bias voltage was gradually increased to $1.5 \mathrm{~V}$. This suggests that the conductance of a single-atom Au contact (which has a conductance close to $\left.G_{0}\right)^{3,4}$ is almost independent of voltage. This is in contrast to several reports ${ }^{2,5,9}$ of currentvoltage $(I-V)$ characteristics measured on stable Au contacts at room temperature (RT), which display clear nonlinearities for voltages larger than $0.1-0.5 \mathrm{~V}$.

In this letter, we present measurements of $I-V$ curves on Au QPCs, which elucidate this apparent disagreement. It is shown that for clean contacts the $I-V$ curves are almost linear in agreement with the conductance histograms. ${ }^{8} \mathrm{We}$ furthermore present self-consistent tight-binding (TB) calculations which support these experimental findings. Only when the sample is not sputtered and annealed do we observe nonlinear $I-V$ curves resembling the previously published curves. ${ }^{2,5,9}$ We can fit the nonlinear curves to a tun-

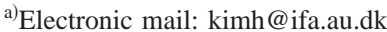

neling model and suggest that these are due to tunneling through a contaminant layer between the electrodes.

Previous measurements of $I-V$ curves on $\mathrm{Au}$ contacts $^{2,5,9}$ were done over a time scale of $0.1-50 \mathrm{~s}$, thus restricting the measurements to contacts which are stable over relatively long time scales. Instead, we use a setup, ${ }^{10}$ whereby an $I-V$ curve can be acquired within only $20 \mu \mathrm{s}$, and transient contact geometries can be studied. The contacts are formed between a polycrystalline Au tip mounted in a scanning tunneling microscope (STM) and a $\mathrm{Au}(110)$ singlecrystal surface under ultra-high-vacuum (UHV) conditions in two different ways: (i) Hard indentations. The tip is first indented 40-100 $\AA$ into the surface and retracted at a speed of $\approx 100 \AA / \mathrm{s}$. The $I-V$ curve is acquired automatically during the break. (ii) Soft indentations. The indentation is stopped the first time the conductance surpasses a preset value of a few $G_{0}$. In a feedback loop, the vertical position of the tip is then continuously adjusted to keep the conductance as stable as possible. When stabilized, a $I-V$ curve is acquired upon request from the user.

In addition, we have investigated how the cleanliness of the $\mathrm{Au}(110)$ sample influences the measured $I-V$ curves by using two different sample preparation techniques: (i) A clean surface (verified by atomic-scale STM imaging) ${ }^{10}$ is produced by sputtering and annealing under UHV conditions. (ii) The sample is rinsed in water and ethanol in air before being placed in an UHV chamber.

Examples of measured $I-V$ curves are shown in Fig. 1. They are almost linear if the sample is sputtered and annealed or the contacts are formed by hard indentations [curves (a) and (b)]. [We can only stabilize contacts formed in soft indentations on a sputtered and annealed surface with a conductance smaller than $4 G_{0}$ by cooling the sample to approximately $-100{ }^{\circ} \mathrm{C}$. $I-V$ curves from such contacts resemble curves (a) and (b) in Fig. 1.] In contrast, nonlinear $I-V$ curves are almost exclusively found when the sample is rinsed in air and the indentations are soft [curve (c)]. 


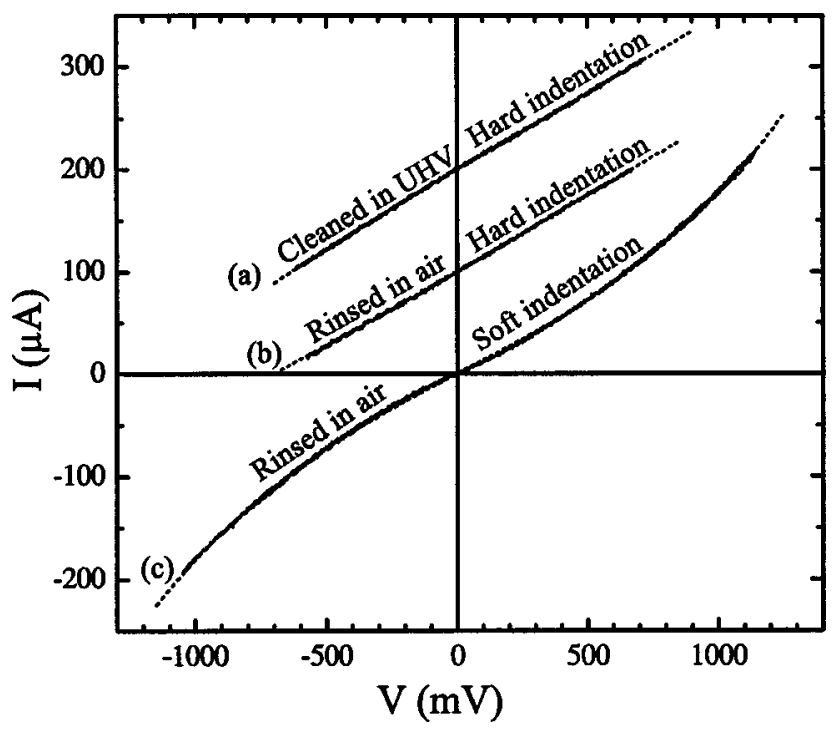

FIG. 1. Examples of measured $I-V$ curves of Au contacts formed at RT using different sample preparation/contact formation techniques. Each curve is acquired during $20 \mu \mathrm{s}$ (a single period of a $50 \mathrm{kHz}$ triangular voltage burst) and consists of 2000 data points. Best fits to Eq. (1) (dashed lines) give the following values of $\left(G, G^{\prime}, G^{\prime \prime}\right)$ in units of $\left(G_{0}, G_{0} / \mathrm{V}, G_{0} / \mathrm{V}^{2}\right)$ : (a) $(1.95,-0.08,0.07)$; (b) $(1.91,0.07,-0.05)$; and (c) $(1.72,-0.02,0.59)$. Individual $I-V$ curves are shifted by $100 \mu \mathrm{A}$ for clarity.

We find that contacts exhibiting nonlinear $I-V$ curves are more robust than their linear counterparts: Reproducible $I-V$ curves can be obtained at voltages up to $\pm 2 \mathrm{~V}$, and the contacts can be stable for hours. In contrast, contacts corresponding to linear $I-V$ curves tend to destabilize for voltages larger than $\approx 0.75 \mathrm{~V}$ (at RT), and contacts with a conductance smaller than $5 G_{0}$ typically break spontaneously within milliseconds.

In general, we find that the $I-V$ curves can be fitted with good accuracy [cf. the dashed lines in Fig. 1] to a third-order polynomial in the applied voltage ${ }^{5}$

$$
I(V)=G V+G^{\prime} V^{2}+G^{\prime \prime} V^{3},
$$

where $G$ is the low-bias conductance, and $G^{\prime}$ is a constant describing the polarity dependence of the current due to asymmetries in the contact region. For most curves $\left|G^{\prime}\right|$ $<0.1 \mathrm{G} / \mathrm{V}$. In the following we will focus on the nonlinear parameter $G^{\prime \prime}$ and its dependence on $G$. Fitted values for $G$ and $G^{\prime \prime}$ based on $I-V$ curves obtained from 148 different contacts are shown in Fig. 2. For $I-V$ curves formed in hard indentations or on a sputtered and annealed sample, the majority of points collapse onto two well-defined regions which have a small third-order term $\left(\left|G^{\prime \prime}\right|<0.2 G_{0} / \mathrm{V}^{2}\right)$ and a lowbias conductance close to $1 G_{0}$ or slightly below $2 G_{0}$. About $80 \%$ of the $I-V$ curves obtained from soft indentations on a rinsed sample have nonlinear terms which exceed $0.2 G_{0} / \mathrm{V}^{2}$ and arbitrary values of $G$. The remaining $20 \%$ are found in the above-mentioned regions.

To further elucidate our experimental findings, we have performed transport calculations for two simple contact geometries on the (110) surface $^{3}$ shown in Fig. 3 (inset). The contacts have either one (a) or two (b) atoms in their smallest cross section. We use a TB model (with $s, p$, and $d$ orbitals) to calculate the electronic structure and current selfconsistently at finite bias. ${ }^{7}$ It is mainly the $6 s$ valence electrons which conduct. In agreement with earlier calculations, ${ }^{7}$

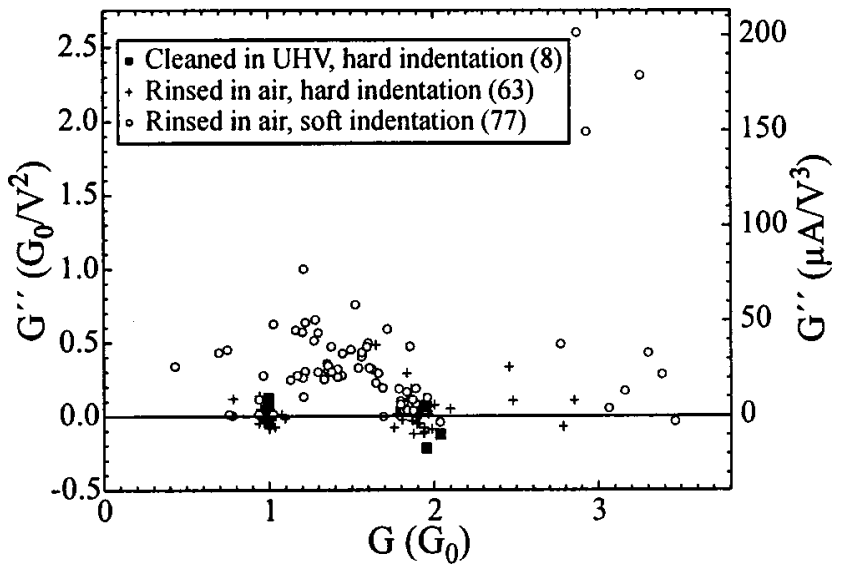

FIG. 2. Nonlinear term $G^{\prime \prime}$ vs low-bias conductance $G$ obtained by fitting measured $I-V$ curves to Eq. (1) for different sample preparation/contact formation techniques. Only fits to nearly polarity independent $I-V$ curves $\left(\left|G^{\prime}\right|<0.05 G / \mathrm{V}\right)$ with small uncertainties of the cubic term $\left(\sigma_{G^{\prime \prime}}\right.$ $\left.<0.01 G / \mathrm{V}^{2}\right)$ are shown. Numbers in parenthesis indicate the number of $I-V$ curves.

we find a conductance of the one-atom contact close to $G_{0}$ : At zero bias the individual channel transmissions $(>0.005)$ are $\{1.00,0.02\}$. (For comparison, the total transmission through a three-atom chain is reduced by 0.04 to $\{0.98\}$, due to loss of tunneling contributions through neighboring atoms. $\left.{ }^{6}\right)$ The transmissions versus energy for the two-atom contact are shown in the panels to the right in Fig. 3 for 0 and $1.5 \mathrm{~V}$. For $0 \mathrm{~V}$ we find a conductance slightly below $2 G_{0}$ which is dominated by two channels with high transmissions $\{0.95,0.91,0.01,0.01\}$. We find that the $I-V$ curve is very linear for the one-atom contact and has a slight down bend for the two-atom contact which is caused mainly by the decrease of one of the main channel transmissions below the Fermi energy $\left(E_{F}\right)$ : Although there are substantial variations

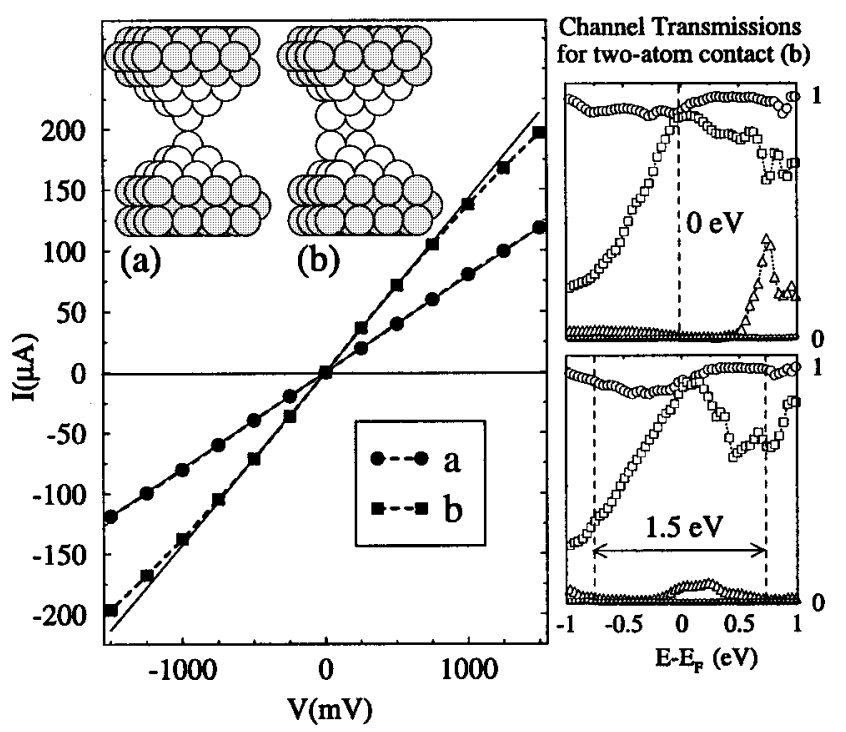

FIG. 3. Left: $I-V$ characteristics calculated using a self-consistent TB model for the (a) one- and (b) two-atom contacts shown in the inset. All interatomic distances are taken to be equal to the bulk value. Solid lines are zero-bias extrapolations corresponding to (a) $G=1.02 G_{0}$ and (b) $G$ $=1.88 G_{0}$. The $I-V$ curve for (b) has $G^{\prime \prime}=-0.07 G_{0} / \mathrm{V}^{2}$. Right: the channel transmissions for (b) (sorted by size and labeled by $\{\bigcirc, \square, \triangle, \circ\}$ ) are shown for 0 and $1.5 \mathrm{~V}$. The vertical dashed lines define the energy window used when calculating the current. 


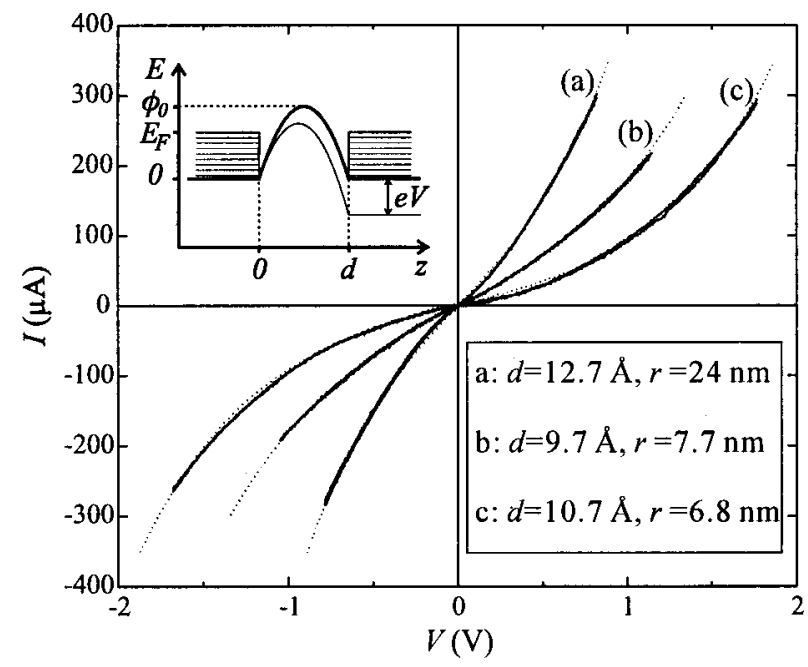

FIG. 4. Solid lines: examples of experimental $I-V$ curves obtained from soft indentaions on a rinsed sample. Dotted lines: fits to the parabolic barrier model sketched in the inset (see Ref. 13) assuming a barrier height $\phi_{0}$ $-E_{F}=1.5 \mathrm{eV}$ and a temperature of $300 \mathrm{~K}$. Fitted values of the barrier thickness $d$ and contact radius $r$ are given in the figure.

in the transmissions, only modest variations are seen in the $I-V$ curve, since the total transmission is found by averaging the sum of individual channel transmissions over the voltage window $\left[E_{F}-e V / 2 ; E_{F}+e V / 2\right]$.

Although Au is a noble metal, it is known that a thin layer of contaminant organic molecules will form spontaneously on the clean surface when exposed to ambient conditions. ${ }^{11}$ When using a sample which has only been rinsed in air, this layer is likely to hinder the formation of a metal-metal contact. ${ }^{11}$ This is especially the case if the contact is formed by soft indentation. In that case, the current will be due to tunneling through an insulating film instead of conduction through an atomic-size metal contact. It is well known $^{12}$ that this leads to $I-V$ curves with a sizable positive value of $G^{\prime \prime}$. Since transmission coefficients in tunneling are orders of magnitudes smaller than those typically encountered in metallic conduction, the contact area needed to reach a conductance of a few $G_{0}$ will be much larger than atomic dimensions. This readily explains the high stability of these contacts, the continuous distribution of $G$ in Fig. 2, and the fact that they can sustain larger voltages than metallic contacts [cf. curve (c) in Fig. 1]. In Fig. 4 we have fitted nonlinear $I-V$ curves to a tunneling model, which has been described in detail elsewhere. ${ }^{13}$ With the barrier thickness $d$ and contact radius $r$ as free parameters, a good agreement is found with reasonable values ( $d \sim 10 \AA, r=6-25 \mathrm{~nm})$.

In hard indentations, the thin contaminant layer is likely to be destroyed, as evidenced by the preference for quantized values of the low-bias conductance in Fig. 2. For the sputtered and annealed sample all $I-V$ curves are almost linear, in perfect agreement with the TB calculations in Fig. 3 and the conductance histograms by Sakai and co-workers. ${ }^{8}$ In contrast, none of the previous measurements of highly nonlinear $I-V$ curves on stable Au contacts at RT were performed on sputtered (and annealed) samples in UHV., ${ }^{2,5,9}$

It has been suggested that the previously observed highly nonlinear $I-V$ curves on Au contacts could be a signature of Luttinger-liquid effects. ${ }^{2,5}$ Our results suggest an alternative and more trivial explanation, namely, that nonlinearities are related to a contamination of the $\mathrm{Au}-\mathrm{Au}$ interface. For clean Au QPCs, our measurements and TB calculations show that the $I-V$ curves are almost linear.

The authors gratefully acknowledge financial support from the Center for Atomic-scale Materials Physics (CAMP) sponsored by the Danish National Research Foundation, and from the Knud Højgaard Foundation.

${ }^{1}$ T. Junno, S.-B. Carlsson, H. Xi, L. Montelius, and L. Samuelson, Appl. Phys. Lett. 72, 548 (1998).

${ }^{2}$ J. I. Pascual, J. Méndez, J. Gómez-Herrero, A. M. Baró, N. García, U. Landman, W. D. Luedtke, E. N. Bogachek, and H.-P. Cheng, Science 267, 1793 (1995).

${ }^{3}$ H. Ohnishi, Y. Kondo, and K. Takayanagi, Nature (London) 395, 780 (1998).

${ }^{4}$ A. I. Yanson, G. R. Bollinger, H. E. van den Brom, N. Agrait, and J. M. van Ruitenbeek, Nature (London) 395, 783 (1998).

${ }^{5}$ J. L. Costa-Krämer, N. García, P. García-Mochales, P. A. Serena, M. I. Marqués, and A. Correia, Phys. Rev. B 55, 5416 (1997); J. L. CostaKrämer, N. García, M. Jonson, I. V. Krive, H. Olin, P. A. Serena, and R. I. Shekter, in Nanoscale Science and Technology, edited by N. García, M. Nieto-Vesperinas, and H. Rohrer, NATO ASI Series (Kluwer, Dordrecht, 1998), p. 1.

${ }^{6}$ J. I. Pascual, J. A. Torres, and J. J. Sáenz, Phys. Rev. B 55, R16029 (1997); A. García-Martín, T. López-Ciudad, J. A. Torres, A. J. Caamaño, J. I. Pascual, and J. J. Sáenz, Ultramicroscopy 73, 199 (1998).

${ }^{7}$ M. Brandbyge, N. Kobayashi, and M. Tsukada, Phys. Rev. B 60, 17064 (1999).

${ }^{8}$ H. Yasuda and A. Sakai, Phys. Rev. B 56, 1069 (1997); K. Itakura, K. Yuki, S. Kurokawa, H. Yasuda, and A. Sakai, ibid. 60, 11163 (1999).

${ }^{9}$ J. Abellán, R. Chicón, and A. Arenas, Surf. Sci. 418, 493 (1998).

${ }^{10}$ K. Hansen, S. K. Nielsen, E. Lægsgaard, I. Stensgaard, and F. Besenbacher, Rev. Sci. Instrum. 71, 1793 (2000)

${ }^{11}$ G. S. Ferguson, M. K. Chaundhury, G. B. Sigal, and G. M. Whitesides, Science 253, 776 (1991); G. M. Whitesides (private communication).

${ }^{12}$ See, e.g., R. Stratton, J. Phys. Chem. Solids 23, 1177 (1962).

${ }^{13} \mathrm{~K}$. Hansen, Ph.D. thesis, University of Aarhus (2000); the model goes beyond the WKB approximation. 